\title{
Exceptionally large migration length of carbon and topographically-facilitated self-limiting molecular beam epitaxial growth of graphene on hexagonal boron nitride
}

\author{
Annette S. Plaut, ${ }^{1, *}$ Ulrich Wurstbauer, ${ }^{2}$ Sheng Wang, ${ }^{3}$ Antonio L. Levy ${ }^{2}$ Lara \\ Fernandes dos Santos, ${ }^{2, \dagger}$ Lei Wang, ${ }^{4}$ Loren N. Pfeiffer, ${ }^{5}$ Kenji Watanabe, ${ }^{6}$ Takashi \\ Taniguchi, ${ }^{6}$ Cory R. Dean, ${ }^{4}$ James Hone, ${ }^{4}$ Aron Pinczuk, ${ }^{2,3}$ and Jorge M. Garcia ${ }^{2,7}$ \\ ${ }^{1}$ Physics and Astronomy, Exeter University, Exeter EX4 $4 Q L$ UK \\ ${ }^{2}$ Department of Physics, Columbia University, New York, NY 10027 USA \\ ${ }^{3}$ Department of Applied Physics, Columbia University, New York, NY 10027 USA \\ ${ }^{4}$ Department of Mechanical Engineering, \\ Columbia University, New York, NY 10027 USA \\ ${ }^{5}$ Electrical Engineering Department, \\ Princeton University, Princeton, NJ 08544 USA \\ ${ }^{6}$ Advanced Materials Laboratory, National Institute \\ for Materials Science, Tsukuba 305-0044 Japan \\ ${ }^{7}$ MBE Lab, IMM-Instituto de Microelectronica de Madrid (CNM-CSIC), \\ E-28760 Tres Cantos Madrid Spain \\ (Dated: November 15, 2016)
}




\begin{abstract}
We demonstrate growth of single-layer graphene (SLG) on hexagonal boron nitride (h-BN) by molecular beam epitaxy (MBE), only limited in area by the finite size of the h-BN flakes. Using atomic force microscopy and micro-Raman spectroscopy, we show that for growth over a wide range of temperatures $\left(500^{\circ} \mathrm{C}-1000^{\circ} \mathrm{C}\right)$ the deposited carbon atoms spill off the edge of the hBN flakes. We attribute this spillage to the very high mobility of the carbon atoms on the BN basal plane, consistent with van der Waals MBE. The h-BN flakes vary in size from $30 \mu \mathrm{m}$ to $100 \mu \mathrm{m}$, thus demonstrating that the migration length of carbon atoms on h-BN is greater than $100 \mu \mathrm{m}$. When sufficient carbon is supplied to compensate for this loss, which is largely due to this fast migration of the carbon atoms to and off the edges of the h-BN flake, we find that the best growth temperature for MBE SLG on h-BN is $\sim 950^{\circ} \mathrm{C}$. Self-limiting graphene growth appears to be facilitated by topographic h-BN surface features: We have thereby grown MBE self-limited SLG on an h-BN ridge. This opens up future avenues for precisely tailored fabrication of nanoand hetero-structures on pre-patterned h-BN surfaces for device applications.
\end{abstract}

\footnotetext{
* Corresponding author: Tel: +44 (0)1392 724199, email: A.S.Plaut@ex.ac.uk

$\dagger$ Present address: Universidade Federal de Santa Catarina, 89065-300, Blumenau, SC Brazil
} 
There has been a huge amount of research into graphene due to its unique electronic, mechanical, optical and thermal properties $[1,2]$ and its vast exploitation potential. Each of its many envisioned applications (e.g. nanocomposites, energy-harvesting, megacapacitors, spintronics, quantum devices ...), presently comes with its own optimal fabrication method. This has led to an explosion in the number of ways to produce graphene, mainly in a drive to increase the area of single-crystal graphene grown. While chemical vapour deposition (CVD) on metal surfaces, produces large-area, multicrystalline, single-layer graphene (SLG) [3-6], if the application requires an insulating substrate then the metal-catalysed CVD-grown SLG requires transfer onto this new substrate after growth. Such transfer steps come with their concomitant problems, such as loss and breakage/cracking of the graphene material, surface/interface contamination and corrugation/wrinkling of the graphene. Molecular beam epitaxy (MBE) [7-26] on the other hand obviates this additional transfer step as MBE growth can be directly onto a diverse range of substrates, both metallic or insulating. Although there have been a few recent reports of CVD growth of graphene directly on dielectrics without metal catalysts [27-34], unlike CVD growth on copper, they do not benefit from the advantage of self-limited growth. In fact a super-linear increase of graphene thickness is even reported after the first monolayer [31].

MBE has a number of further advantages: in-situ surface-science characterisation [35], to enhance our understanding of the growth mechanisms; large area graphene, in principle only limited in size by the dimensions of the MBE growth chamber and, most importantly, the potential to grow graphene-semiconductor heterostructures - so-called van der Waals heterostructures [36].

The choice of an appropriate insulating substrate is crucial for growing high-quality graphene by MBE. Aside from its dielectric properties, the major requirements for such a substrate are flatness on an atomic scale, inertness in the presence of atomic carbon and high temperature stability. Hexagonal boron nitride (h-BN), with its similar atomic structure to graphene, thus stands out as an ideal substrate for epitaxial MBE growth of graphene $[15,22,25,26]$. Not only that, but it is also an ideal candidate as a spacer in van der Waals heterostructures [36].

In this paper we report on an investigation into the effect of temperature on the MBE growth of a high-quality continuous graphene on h-BN, only limited in size by the lack of availability of larger h-BN single crystals. We find that MBE growth of graphene on h-BN 
is dictated by the high mobility of the carbon atoms on the h-BN surface, in a manner that is consistent with van der Waals epitaxy [37]. We observe that the carbon atoms spill off the edge of the h-BN flakes, which shows that the carbon atoms have a migration length greater than $100 \mu \mathrm{m}$ on h-BN. This results, as we demonstrate using Raman spectroscopy and atomic-force microscopy (AFM), in only a small fraction of the evaporated carbon actually remaining on the h-BN flakes. We also show the key role in the growth process that is played by topographic features on the h-BN surface, which leads to self-limiting MBE SLG growth.

The graphene layers are grown in an ultra-high vacuum (UHV) environment inside a home-built molecular beam system $[14,15,38]$. Multiple h-BN substrate flakes are obtained by micro-mechanical cleavage of $\mathrm{h}-\mathrm{BN}$ and are deposited on a rectangular $(25 \mathrm{~mm} \times 7 \mathrm{~mm})$ support wafer. The h-BN flakes vary in size from $30 \mu \mathrm{m}$ to $100 \mu \mathrm{m}$. For this study h-BN flakes were chosen due to their dark blue color under white light illumination through an optical microscope, indicating that their thickness was only $10 \mathrm{~nm}-15 \mathrm{~nm}$ [39]. Prior to growth, a surface cleaning treatment is performed in-situ by annealing the substrate at temperatures $\geq 400^{\circ} \mathrm{C}$ in UHV for a few hours. This procedure removes any residues of the Scotch-Tape exfoliation process. We have employed two different support wafers: c-plane (0001) oriented sapphire and $\mathrm{SiO}_{2} / \mathrm{Si}(001)$, comprising of a $300 \mathrm{~nm}$-thick layer of $\mathrm{SiO}_{2}$ on $\mathrm{Si}$. Thermal decomposition limits the maximum growth temperature for $\mathrm{SiO}_{2} / \mathrm{Si}$ to $930^{\circ} \mathrm{C}[40,41]$, while sapphire melts only at $\sim 2000^{\circ} \mathrm{C}$.

The amount of delivered carbon to the h-BN flakes is carefully estimated using an ex-situ calibration of the cell flux versus operating power and the position of the h-BN flake on the support wafer. More details can be found elsewhere [15].

The topography of the grown layers was examined by tapping-mode AFM under ambient conditions. Micro-Raman measurements were obtained using a Renishaw inVia Raman microscope equipped with an $\mathrm{x}-\mathrm{y}-\mathrm{z}$ stage, excited by a $532 \mathrm{~nm}$ laser focused to a $\lesssim 1 \mu \mathrm{m}$ diameter spot at an incident power of $\lesssim 3 \mathrm{~mW}$. A broad optical emission background, which appears after the surface cleaning treatment, underpins all the Raman spectra taken on h-BN. This background is well described by a polynomial function and has been subtracted from the raw data [15].

Raman spectroscopy is a powerful tool to study graphene as it is sensitive to crystal quality, in particular the crystallinity and any strain and defects in the graphene layers. Raman peaks are observed both from the graphene itself and its underlying substrate and 

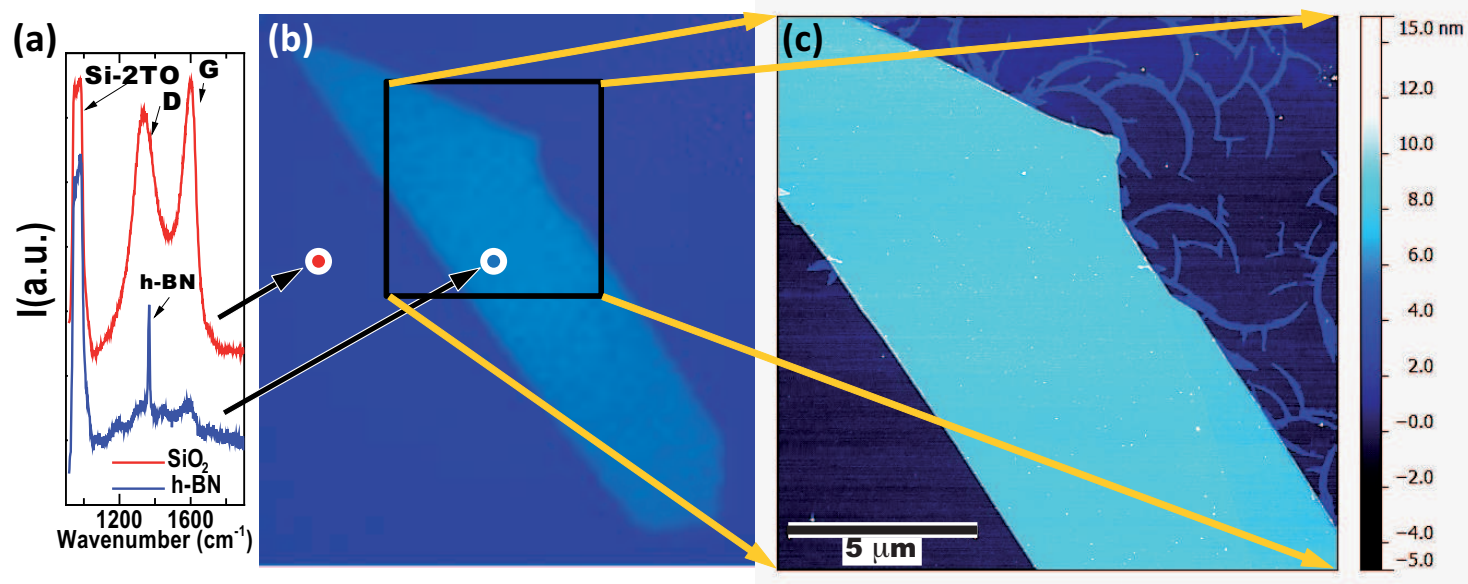

FIG. 1. Graphitic layers grown by MBE at a growth temperature of $T_{s}=500^{\circ} \mathrm{C}$, on an h-BN flake on a $\mathrm{SiO}_{2} / \mathrm{Si}$ support wafer. (a) Raman spectra taken from the middle of the h-BN flake (blue) and from a region adjacent to it on the $\mathrm{SiO}_{2} / \mathrm{Si}$ support wafer (red). The spectra have been offset for clarity. (b) An optical microscope image of the graphitic layers on the h-BN flake and on the support wafer with the locations, where the spectra in (a) were taken, indicated. (c) A $15 \times 15 \mu \mathrm{m}^{2}$ atomic force microscopy image of the region boxed in black in (b).

support wafer. The silicon support wafer produces Raman lines at energy shifts of $520 \mathrm{~cm}^{-1}$, $\sim 1000 \mathrm{~cm}^{-1}$ and $1450 \mathrm{~cm}^{-1}$, corresponding to the first order transverse optical phonon peak (Si-1TO), the Si-2TO and Si-3TO, respectively, while the $\mathrm{E}_{2 g}$ optical phonon from the h-BN substrate manifests as a sharp Raman feature at $1370 \mathrm{~cm}^{-1}$ [42]. The characteristic Raman signatures of the graphene itself are the D peak, at an energy shift of $1350 \mathrm{~cm}^{-1}$, the $\mathrm{G}$ $\left(\mathrm{E}_{2 g}\right)$ peak at $1590 \mathrm{~cm}^{-1}$, the D' line at $1620 \mathrm{~cm}^{-1}$ and the $2 \mathrm{D}$, or G', peak at $2690 \mathrm{~cm}^{-1}$. Although the appearance of the D peak signifies the existence of hexagonal carbon rings, it requires the presence of disorder, such as edges or atomic defects to be activated. The $2 \mathrm{D}$ mode is the most sensitive to the quality of the crystal, strain, doping and the alignment between the graphene and h-BN atomic lattices. Therefore an observation of a intense and narrow 2D mode is a strong indication of the high crystalline quality of the grown layers, although at very low misalignment angles $\left(<2^{\circ}\right)$ the $2 \mathrm{D}$ peak has been shown to broaden and to change from Lorentzian to Gaussian in shape [43].

Fig. 1a shows Raman spectra from two adjacent regions - from the graphitic layer grown on an h-BN flake (blue) and from graphitic layers grown on the $\mathrm{SiO}_{2} / \mathrm{Si}$ support wafer just next to that h-bN flake (red). The growth temperature $\left(T_{s}\right)$ was $500^{\circ} \mathrm{C}$ and the graphitic 
layers were grown for $40.3 \mathrm{~min}$. Thus the amount of carbon delivered to this sample is estimated to be $4.8 \mathrm{~nm}$, which is equivalent to $\sim 13.7$ graphene monolayers (ML) [15]. The location of where these Raman measurements were taken is shown in Fig. 1b as two dots with colors corresponding to their respective spectra.

The two spectra of Fig. 1a have a number of Raman peaks in common: The Si-2TO and the graphene D and G peaks. The spectrum from the graphitic layer on the h-BN flake however contains two extra peaks - the h-BN optical phonon and the faint indication of the Si-3TO peak. The latter is only visible in this spectrum, due to the absence of the Raman intensity between the graphene D and G lines, which is seen in the spectrum from the graphitic layers grown on the $\mathrm{SiO}_{2} / \mathrm{Si}$ support wafer, which originates from the presence of disordered $\mathrm{sp}^{2}$ carbon bonds [14,44-48]. Note that the 2 TO phonon resonance associated with the silicon support wafer is equally intense in both spectra, demonstrating the high transparency of the h-BN flake.

Surprisingly, the Raman spectra from these two adjacent regions differ remarkably in intensity, suggesting a completely distinct number of graphitic layers present on and off the h-BN flake: A quantitative estimation [49] of the number of graphitic layers at each location is possible by analyzing the ratio of the integrated intensity of the $\mathrm{G}$ line to that of the silicon 2TO phonon line $-A(\mathrm{G}) / A(\mathrm{Si}-2 \mathrm{TO})$ - assuming that the h-BN flake is transparent. The presence, in the spectrum taken off the h-BN flake, of significant Raman intensity between the graphene D and $G$ lines hinders any very accurate determination of the area of the G-peak from this spectrum, but even so, the $G$ peak is sufficiently intense for this only marginally to affect the result of such analysis.

For comparison, we also measured and derived $A(\mathrm{G}) / A(\mathrm{Si}-2 \mathrm{TO})$ from the Raman spectra of graphene flakes, fabricated by micromechanical cleavage of highly-oriented pyrolytic graphite (HOPG), which we have also deposited on a substrate of $300 \mathrm{~nm}$-thick $\mathrm{SiO}_{2}$ on Si. These latter we have compared to data produced following the procedure described in Ref. 50, where comparison was made to the Si-1TO line instead. We found [49] an identical trend in both $A(\mathrm{G}) / A(\mathrm{Si}-1 \mathrm{TO})$ and $A(\mathrm{G}) / A(\mathrm{Si}-2 \mathrm{TO})$ with the number of monolayers, i.e. they both increase stepwise with exfoliated graphene flake number. The only difference is the actual value of their intensity ratios. We have assigned the number of graphene layers to the individual discrete steps by the direct comparison of our $A(\mathrm{G}) / A(\mathrm{Si}-1 \mathrm{TO})$ data with Fig. 2 of Ref. 50. SLG was also identified by the single-Lorentzian lineshape of its 2D Raman 
line and the significantly greater Raman intensity of this $2 \mathrm{D}$ line compared to that of its $\mathrm{G}$ peak [49]. Using this information we have compared the ratio $A(\mathrm{G}) / A(\mathrm{Si}-2 \mathrm{TO})$ from spectra taken on a h-BN flake and on $\mathrm{SiO}_{2} / \mathrm{Si}$ and have hence derived estimates for the thickness of the graphitic layers in each case: $0.25 \mathrm{ML}$ or less on the h-BN and at least $5 \mathrm{ML}$, most probably greater than $6 \mathrm{ML}$, directly on the $\mathrm{SiO}_{2} / \mathrm{Si}$ support wafer, which implies that the fraction of the carbon that remains on the h-BN flake is only $3 \%-5 \%$.

Fig. 1c is an AFM image of the area marked by the black box in Fig. 1b. This AFM image of the same flake shows large dendritic structures (height $\sim 3.5 \mathrm{~nm}-4 \mathrm{~nm}$ ) growing from the h-BN upper-right edge. Remarkably, only one edge acts as a preferential nucleation centre for these dendritic structures, probably reflecting a chemical anisotropy in the h-BN edges. Since our h-BN flakes are all randomly oriented on the support substrate, we find that these dendritic structures are oriented in different directions at each individual location of an h-BN flake across the support wafer, reflecting that flake's orientation. Calculations in the literature [22] indicate that the sticking coefficient of atomic carbon to h-BN is small and that the energy barrier to diffusion of the carbon ad-atoms on the h-BN surface is even lower than the energy barrier to their desorption. The sticking coefficient is defined as the ratio of the rate that adsorbate atoms, or molecules, adsorb, to the rate at which atoms/molecules impinge upon that surface. We however are not actually measuring the sticking coefficient per se, but instead we are measuring the fraction of carbon atoms remaining on the h-BN surface. From our observation of the spillage of the carbon atoms off the h-BN flakes in Fig. 1c, we can further deduce that the remaining carbon atoms have a migration length on h-BN larger than the finite size of the flakes $-30 \mu \mathrm{m}-100 \mu \mathrm{m}-$ (even at this low $T_{s}$ ). The migration length is the distance travelled by the incoming carbon atoms on the surface of h-BN before incorporation into the atomic lattice. The migration length of atomic carbon has not been previously measured and the large value that we find is indicative of high atomic mobility which is characteristic of van der Waals molecular beam epitaxial (vdW-MBE) growth [37].

We thus conclude that during vdW-MBE growth of graphene on h-BN flakes, it is therefore necessary, at all growth temperatures, to supply excess carbon atoms during growth to compensate for the fraction that spills off the h-BN flake [22, 25, 26]. We have investigated the vdW-MBE growth of graphene on h-BN as a function of increasing growth temperature, in order to ascertain the best possible growth temperature for successful monolayer vdW- 


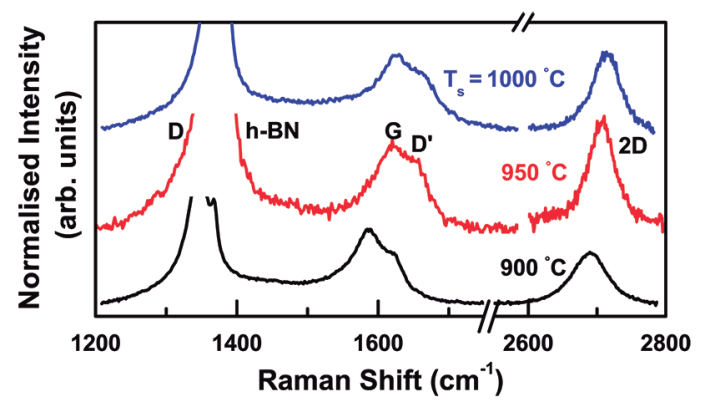

FIG. 2. Raman spectra of vdW-MBE grown SLG graphene at various growth temperatures on an h-BN flake on a sapphire support wafer. At each growth temperature an h-BN flake has been chosen within a narrow range of locations $(<2.4 \mathrm{~mm}$ apart) on the support wafer to ensure that similar amounts of carbon were delivered for each of the spectra. All other growth conditions were identical for all the three growth temperatures. The spectra have been offset for clarity and their intensities have been normalised so that the G-peak intensity is constant across all 3 spectra.

MBE growth of high-quality graphene on h-BN. Since $\mathrm{SiO}_{2} / \mathrm{Si}$ thermally decomposes above $930^{\circ} \mathrm{C}[40,41]$ we have employed a sapphire support wafer instead.

Fig. 2 focuses in on Raman spectra in the range of growth temperatures $T_{s}=900^{\circ} \mathrm{C}-$ $1000^{\circ} \mathrm{C}$. The constraints of our MBE system limit us to a maximum growth temperature of about $1000^{\circ} \mathrm{C}$. The graphene was grown under identical growth conditions (growth time $=65.0$ minutes) except for the location of the h-BN substrate on the support wafer, which was kept within a narrow range $(<2.4 \mathrm{~mm}$ apart $)$ to ensure that a similar amount of carbon was delivered in each case. Thus the amount of carbon supplied to these samples is estimated to be $0.69 \mathrm{~nm}-0.85 \mathrm{~nm}(\sim 1.96-2.42 \mathrm{ML})$. We have successfully line-fitted each Raman peak of the spectra of Fig. 2 with a Lorentzian function. Taking into account that only a tiny fraction of the carbon deposited on the h-BN is adsorbed and incorporated into the epitaxial graphene and that the 2D Raman peak in the spectra for all 3 temperatures shown in Fig. 2 can be fitted by a single narrow Lorentzian function, we can be fairly certain that we are measuring single-layer graphene in all 3 cases, although only for the 2 highest temperatures is the 2D line's peak intensity significantly greater than that of the G Raman peak. It is interesting to note that the amount of carbon that remains on the h-BN surface increases with growth temperature: We obtain very a low coverage of graphitic carbon when we grow at $500^{\circ} \mathrm{C}$ on h-BN flakes on a silicon support wafers (Fig. 1), graphene nanodomains 
at a growth temperature of $930^{\circ} \mathrm{C}$ on h-BN flakes on silicon support wafers (not shown), but continuous SLG at $T_{s}=900^{\circ} \mathrm{C}-1000^{\circ}$ on h-BN flakes on sapphire support wafers (Figs. 2 and 3). Thus we observe that that carbon appears to stick better to the h-BN with increasing temperature and when the h-BN flakes are supported on sapphire rather than silicon. From detailed Raman spectral analysis we conclude that growth at $T_{s}=950^{\circ} \mathrm{C}$ produces the highest quality graphene: $T_{s}=950^{\circ} \mathrm{C}$ gives the highest ratio of the peak intensity of the graphene $2 \mathrm{D}$ line to that of the $\mathrm{G}$ line, $I(2 \mathrm{D}) / I(\mathrm{G})=1.38$, and the lowest $I(\mathrm{D}) / I(\mathrm{G})=1.59$. In fact, the absolute value of $\mathrm{I}(\mathrm{D})$ actually attains its minimum value at $T_{s}=950^{\circ} \mathrm{C}$. The origin of the $\mathrm{D}$ line is discussed in detail below. The linewidth of the $2 \mathrm{D}$ peak, $\Delta \omega_{2 \mathrm{D}}$, at $T_{s}=950^{\circ} \mathrm{C}$ is $56 \mathrm{~cm}^{-1}$, while that of the $\mathrm{G}$ peak, $\Delta \omega_{\mathrm{G}}$, is $63 \mathrm{~cm}^{-1}$. While at $T_{s}=1000^{\circ} \mathrm{C}$, $\Delta \omega_{2 \mathrm{D}}=54 \mathrm{~cm}^{-1}$ and $\Delta \omega_{\mathrm{G}}=57 \mathrm{~cm}^{-1}$. However the 2D line's height to width ratio peaks at $950^{\circ} \mathrm{C}$. In addition the energy positions of our $\mathrm{G}$ and $2 \mathrm{D}$ lines do not give any indication of any strain being present in our graphene $[51,52]$. Although we are constrained, by our MBE system, to a maximum growth temperature of about $1000^{\circ} \mathrm{C}$, this analysis points to growth temperatures above $1000^{\circ} \mathrm{C}$ not necessarily leading to higher quality MBE grown graphene. Despite claims in the literature that growth temperatures above $\sim 1000^{\circ} \mathrm{C}$ are required to provide the necessary mobility of the carbon on the growth surface [25], the evidence from Fig. 1 is that the carbon atoms are already extremely mobile on the h-BN surface even at growth temperatures as low as $500^{\circ} \mathrm{C}$.

Spectra such as those shown in Fig. 2 are typical of vdW-MBE grown graphene in that they contain a fairly intense and sharp Raman D line. The presence of an intense, sharp Raman D lines is indicative of the existence of edges, as in the spectra reported from graphene nanoribbons (GNRs) fabricated from etched exfoliated pristine graphene [53, 54]. The spectra of Fig. 2 exhibit striking similarities to those of such GNRs. Our vdW-MBE-grown graphene contains numerous grain boundaries which we believe are responsible for the breaking of wavevector conservation in our inelastic light-scattering experiments, leading to the presence of a D peak in our Raman spectra. This interpretation is supported by our analysis of $I(\mathrm{D}) / I\left(\mathrm{D}^{\prime}\right)$ : It has been shown that $I(\mathrm{D}) / I\left(\mathrm{D}^{\prime}\right)$ enables identification of the type of lattice defects present in graphene [55]. $I(\mathrm{D}) / I\left(\mathrm{D}^{\prime}\right)=3.76$ for our vdW-MBE grown graphene at $T_{s}=950^{\circ} \mathrm{C}$, which indicates that it is indeed grain boundaries that are responsible for the breaking of translational symmetry required to observe the D and D' Raman lines in this case. 


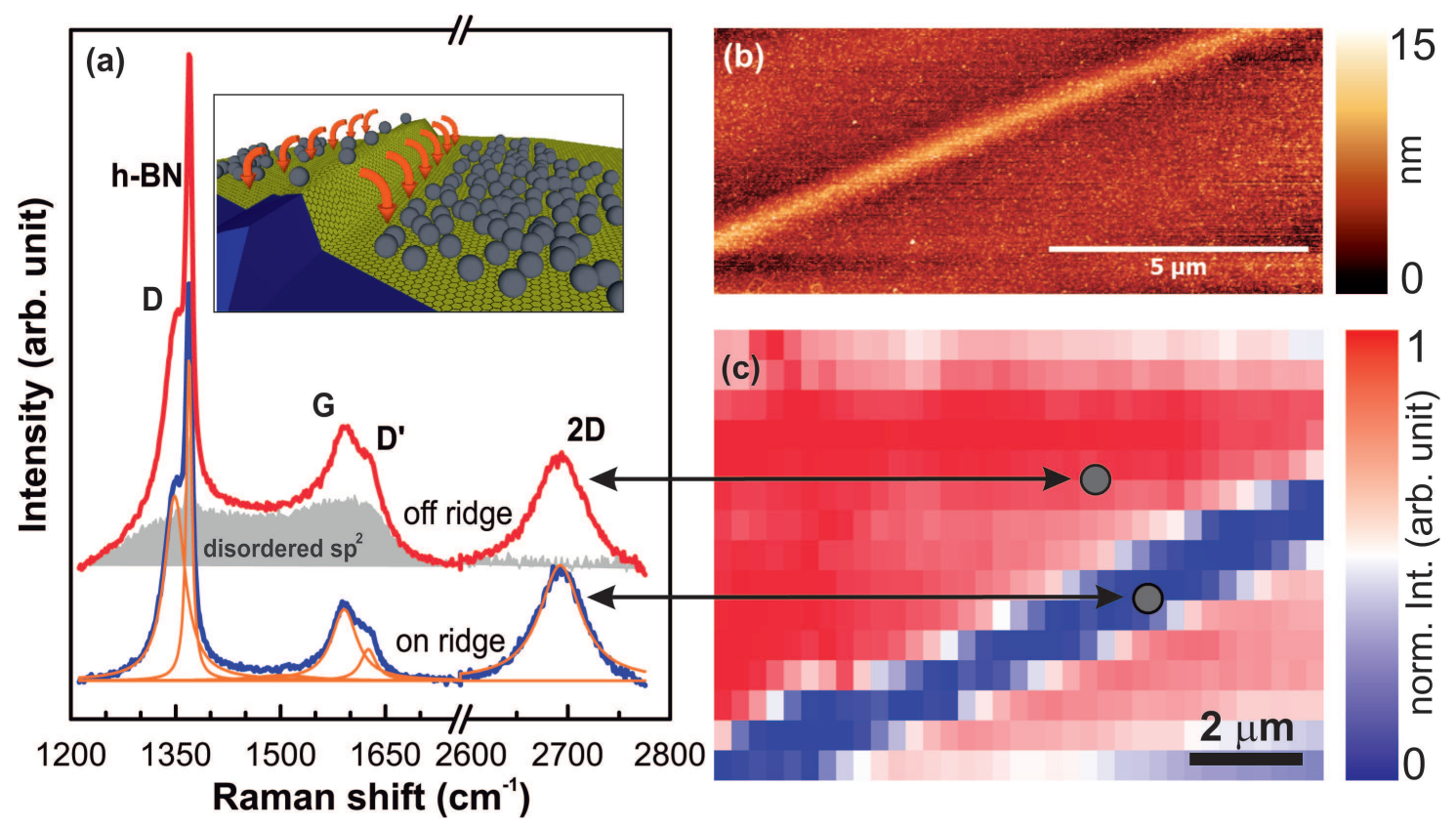

FIG. 3. vdW-MBE graphene grown at $T_{s}=1000^{\circ} \mathrm{C}$ on an h-BN flake containing a top surface ridge, on a sapphire support wafer, in an excess of carbon. (a) Raman spectra taken 'on' (blue) and 'off' (red) the ridge. The grey shaded area corresponds to the calculated difference between the 'off ridge' and 'on ridge' spectra and indicates the absence of disordered $\mathrm{sp}^{2}$ carbon bonds on the ridge. The yellow trace associated with the 'on-ridge' (blue) spectrum is the result of lineshape analysis of the individual Raman lines. The spectra have been offset for clarity. The inset shows an artistic impression of the growth process after formation of the initial SLG. (b) A $\sim 5 \times 12 \mu \mathrm{m}^{2}$ AFM measurement showing the ridge which has a height of $\sim 6 \mathrm{~nm}$ and a width of $\sim 750 \mathrm{~nm}$. (c) A Raman map of the integrated intensity between $1422 \mathrm{~cm}^{-1}$ and $1524 \mathrm{~cm}^{-1}$. The absence of disordered $\mathrm{sp}^{2}$ carbon bonds in the 'on ridge' spectra is clearly shown by the blue color associated with the ridge itself. The grey dots are the locations where the spectra in (a) were taken.

In Fig. 3 we show results from graphene grown by vdW-MBE at a $T_{s}=1000^{\circ} \mathrm{C}$ on an h-BN flake $(\sim 24 \mu \mathrm{m} \times 15 \mu \mathrm{m}$ in area) on a sapphire support wafer, where the h-BN flake has a ridge in its top surface. $8.4 \mathrm{~nm}(\sim 24 \mathrm{ML})$ of carbon were supplied during growth for a growth time of 65.0 minutes. The ridge itself was found by AFM - Fig. 3b - to have a height of of $\sim 6 \mathrm{~nm}$ and a width of $\sim 750 \mathrm{~nm}$. A Raman spectrum taken 'on ridge' (blue spectrum in Fig. 3a) exhibits the characteristic features of SLG. The peaks are well resolved, narrow and separated from each other with no light-scattering intensity 
between them, demonstrating the high quality of the SLG grown. This identification of SLG is corroborated by our detailed spectral analysis described below. Meanwhile a spectrum taken 'off ridge' (red trace in Fig. 3a) displays, together with narrow and well resolved peaks, an additional broad Raman scattering continuum extending from $\sim 1300 \mathrm{~cm}^{-1}$ to beyond $1600 \mathrm{~cm}^{-1}$. The numerical difference of the absolute intensities of these two spectra is shown, shaded grey, in Fig. 3a. This broad Raman signal is a measure of the amount of disordered, i.e. non-planar, carbon $\mathrm{sp}^{2}$ bonds present (and is also apparent in the Raman spectrum taken adjacent to the h-BN flake in Fig. 1a) [14, 44-48]. This integrated intensity between the h-BN and the $\mathrm{G}$ resonances (from $1422 \mathrm{~cm}^{-1}$ to $1524 \mathrm{~cm}^{-1}$ ) has been normalized and mapped in Fig. 3c over a $10.8 \mu \mathrm{m} \times 8.5 \mu \mathrm{m}$ area. We have observed an accurate spatial correlation between the high-quality graphene ribbon (shown blue in Fig. 3c) and the ridge topography observed by AFM (Fig. 3b). Strikingly the 2D peak 'on' and 'off' the ridge is identical, as is clearly visible in Fig. 3a, suggesting the presence in both cases of SLG, but the 'on-ridge' spectra do not suffer from the presence of disordered $\mathrm{sp}^{2}$ carbon bonds.

In fact this 2D Raman resonance remains unchanged over the whole measured area in Fig. 3c, irrespective of the presence of the ridge. Since the other Raman peaks are also similar in the 'on' and 'off' spectra (apart from the broad underlying continuum due to disordered $\mathrm{sp}^{2}$ bonds), we speculate that initially homogeneous, uniform SLG of high crystal quality is formed over the entire h-BN flake. The energy positions of our G and 2D Raman lines do not give any indication of any strain being present, neither 'on ridge' nor 'off ridge' [51, 52]. Additional carbon atoms impinging on the substrate diffuse from 'on ridge' to 'off ridge' areas where they form non-planar carbon bonds. Therefore high-crystal-quality self-limited SLG remains only in the 'on ridge' region. This formation process is schematically depicted in the inset to Fig. 3a. Migration of atoms on the surface of a substrate during epitaxial growth is governed by the surface chemical potential [56]. Variations in this chemical potential are directly proportional to surface curvature. The resulting net flow of atoms is towards regions of lower chemical potential - concave surfaces - from regions of higher chemical potential - convex or flat surfaces [56], as depicted in the inset to Fig. 3a. The high-quality vdWMBE grown single-layer graphene on the h-BN ridge has an overall area of $>10 \mu \mathrm{m}^{2}$. Thus growth on such h-BN topographical features may open up avenues, in future, for fabrication of nanostructured devices grown on pre-patterned h-BN surfaces.

Detailed Raman spectral analysis of the 'on ridge' self-limited SLG spectrum (Fig. 3a 
- blue) has been undertaken by successfully fitting the spectra with a Lorentzian function for each Raman peak. The results of such fitting procedures are displayed in yellow in Fig. 3a and they clearly accurately reproduce the measured spectrum. This analysis provides strong evidence for both the single-layer graphene nature and the high crystal quality of the graphene on the ridge: the $2 \mathrm{D}$ peak is best described by a single Lorentzian, the linewidths of the $2 \mathrm{D}\left(46.8 \mathrm{~cm}^{-1}\right)$ and $\mathrm{G}\left(55.0 \mathrm{~cm}^{-1}\right)$ lines are narrow and $I(2 \mathrm{D}) / I(\mathrm{G})=1.44$. This relatively high $I(2 \mathrm{D}) / I(\mathrm{G})$ ratio rules out the possibility that we are observing turbostratic [57] rather than single-layer graphene on the ridge, as for turbostratic multilayer graphene the value of this $I(2 \mathrm{D}) / I(\mathrm{G})$ ratio would be about half that which we measure for our SLG. Raman peak broadening can arise from the presence of charge carriers in the grown SLG [58, 59], from strain induced by distortion of the carbon lattice $[60,61]$ and from a small misalignment between the h-BN and graphene atomic lattices [43]. However in the latter case, as mentioned previously, the $2 \mathrm{D}$ peak would also change from a Lorentzian to a Gaussian in shape. As our 2D line is successfully fitted by an Lorentzian function, we can rule out such misalignment of the atomic lattices as the cause of any broadening of our 2D Raman line. Thus in our case, doping due to unintentional incorporation of nitrogen/boron atoms from the h-BN substrate or non-carbon atoms from any residual gas in the growth chamber, is the most likely cause of any line broadening $[62,63]$.

For our on-ridge self-limited SLG $I(\mathrm{D}) / I(\mathrm{G})=2.33$, which indicates that our SLG, although continuous, is not devoid of defects. However $I(\mathrm{D}) / I\left(\mathrm{D}^{\prime}\right)=7.4$, which indicates that vacancy-like defects are mainly responsible for the breaking of translational symmetry required to observe the $\mathrm{D}$ and $\mathrm{D}$ ' Raman lines in the case of this continuous self-limited SLG on the h-BN ridge [55].

In summary, our results show the successful growth of continuous SLG on h-BN by MBE, only limited in area by the finite size of the h-BN flake. We find that the best possible growth temperature for such MBE SLG on h-BN is $\sim 950^{\circ} \mathrm{C}$. We observe that, at all growth temperatures $\left(500^{\circ} \mathrm{C}-1000^{\circ} \mathrm{C}\right)$, only a small fraction $(3 \%-5 \%)$ of the evaporated carbon remains on the h-BN flake. We attribute this spillage, of the evaporated carbon atoms off the h-BN flakes, to the very high mobility and long migration length (greater than $100 \mu \mathrm{m}$ ) of the carbon atoms on the BN basal plane, consistent with van der Waals MBE. We have hence grown, at $T_{s}=1000^{\circ} \mathrm{C}$, in the presence of an excess of evaporated carbon atoms to compensate for the loss due to spillage, high-quality, continuous SLG which we have shown 
to be self-limiting on an h-BN ridge. Such self-limiting vdW-MBE SLG growth may open up avenues, in future, for the precisely tailored fabrication of nano- and hetero-structures on pre-patterned h-BN surfaces for device applications.

This work is supported by ONR (N000140610138 and Graphene MURI), AFOSR (FA9550-11-1-0010), EFRC Center for Re-Defining Photovoltaic Efficiency through Molecule Scale Control (award DE-SC0001085), NSF (CHE-0641523), NYSTAR and Spanish Government (AIC-B-2011-0806, MAT2014-54231, MAT2015-67021-R).

[1] For a review see: Geim AK, Novoselov KS. The rise of graphene. Nat Mater 2007;6:183-191.

[2] For a review see: Bonaccorso F, Sun Z, Hasan T, Ferrari AC. Graphene photonics and optoelectronics. Nat Photon 2010;4:611-622.

[3] Li, X, Cai W, An J, Kim S, Nah J, Yang D. Large-area synthesis of high-quality and uniform graphene films on copper foils. Science 2009;324:1312-1314.

[4] Kim KS, Zhao Y, Jang H, Lee SY, Kim JM, Kim K.S, et al. Large-scale pattern growth of graphene films for stretchable transparent electrodes. Nature 2009;457:706-710.

[5] Bae S, Kim H, Lee Y, Xu X, Park J-S, Zheng Y, et al. Roll-to-roll production of 30-inch graphene films for transparent electrodes. Nat Nanotechnol 2010;5:574-578.

[6] Hao Y, Bharathi MS, Wang L, Lui Y, Chen H, Nie S, et al. The role of surface oxygen in the growth of large single-crystal graphene on copper Science 2013;720:720-723.

[7] Park J, Mitchel WC, Grazulis L, Smith HE, Eyink KG, Boeckl JJ, et al. Epitaxial graphene growth by carbon molecular beam epitaxy (CMBE). Adv Mater 2010;22:4140-4145.

[8] Moreau E, Godey S, Ferrer FJ, Vignaud D, Wallart X, Avila J, et al. Graphene growth by molecular beam epitaxy on the carbon-face of SiC. Appl Phys Lett 2010; 97:241907.

[9] Jerng SK, Yu DS, Kim YS, Ryou J, Hong S, Kim C, et al. Nanocrystalline graphite growth on sapphire by carbon molecular beam epitaxy. J Phys Chem C 2011;115:4491-4494.

[10] Lippert G, Dabrowski J, Lemme M, Marcus C, Seifarth O, Lupina G. Direct graphene growth on insulator. Phys Status Solidi B 2011;248:2619-2622.

[11] Tang J, Kang CY, Li LM, Yan WS, Wei SQ, Xu PS. Graphene films grown on Si substrate via direct deposition of solid-state carbon atoms. Physica E 2011;43:1415-1418.

[12] Kang C-Y, Tang J, Lui Z-L, Li L-M, Yan W-S, Wei W-S, et al. Growth of few-layer graphene 
on sapphire substrates by directly depositing carbon atoms. Chin Phys Lett 2011;28:1181011-118101-3.

[13] Lui Z, Tang J, Kang C, Zou C, Yan W, Xu P. Effect of substrate temperature on few-layer graphene grown on $\mathrm{Al}_{2} \mathrm{O}_{3}\left(\begin{array}{llll}0 & 0 & 0 & 1\end{array}\right)$ via direct carbon atoms deposition. Solid State Commun $2012 ; 152: 960-963$.

[14] Wurstbauer U, Schiros T, Jaye C, Plaut AS, He R, Rigosi A, et al. Molecular beam growth of graphene nanocrystals on dielectric substrates. Carbon 2012;50: 4822-4829.

[15] Garcia JM, Wurstbauer U, Levy A, Pfeiffer LN, Pinczuk A, Plaut AS, et al. Graphene growth on h-BN by molecular beam epitaxy. Solid State Commun 2012;152:975-978.

[16] Lin M-Y, Guo W-C, Wu M-H, Wang P-Y, Lui T-H, Pao C-W, et al. Low-temperature grown graphene films by using molecular beam epitaxy. Appl Phys Lett 2012;101:221911-1-221911-4.

[17] Lippert G, Dabrowski J, Yamamoto Y, Herziger F, Maultzsch J, Lemme MC, et al. Molecular beam growth of micrometer-size graphene on mica. Carbon 2013;52:40-48.

[18] Oliveira Jr M.H, Schumann T, Gargallo-Caballero R, Fromm F, Seyller T, Ramsteiner M, et al. Mono- and few-layer nanocrystalline graphene grown on $\mathrm{Al}_{2} \mathrm{O}_{3}\left(\begin{array}{lll}0 & 0 & 0\end{array}\right)$ by molecular beam epitaxy. Carbon 2013;56:339-350.

[19] Kiraly B, Iski EV, Mannix AJ, Fisher BL, Hersam MC, Guisinger NP. Solid-source growth and atomic-scale characterization of graphene on $\mathrm{Ag}(111)$. Nat Commun 2013;4:2804-1-2804-7.

[20] Schumann T, Dubslaff M, Oliveira Jr MH, Hanke M, Fromm F, Seyller T, et al. Structural investigation of nanocrystalline graphene grown on $(6 \sqrt{3} \times 6 \sqrt{3}) \mathrm{R} 30^{\circ}$-reconstructed SiC surfaces by molecular beam epitaxy. New J Phys 2013;15:123034-1-123034-16.

[21] Wang S, Fernandos dos Santos L, Wurstbauer U, Wang L, Pfeiffer LN, Hone J, et al. Singleand bi-layer graphene grown on sapphire by molecular beam epitaxy. Solid State Commun $2014 ; 189: 15-20$.

[22] Dabrowski J, Lippert G, Schroeder T, Lupina G. Role of defects in the process of graphene growth on hexagonal boron nitride from atomic carbon. Appl Phys Lett 2014;105:191610-1$191610-5$.

[23] Schumann T, Lopes JMJ, Wofford JM, Oliveira Jr MH, Dubslaff M, Hanke M, et al. The impact of substrate selection for the controlled growth of graphene by molecular beam epitaxy. J Cryst Growth 2015;425:274-278.

[24] Zuo Z, Xu Z, Zheng R, Khanaki A, Zheng J-G, Lui J. In-Situ Epitaxial Growth of 
Graphene/h-BN van der Waals heterostructures by molecular beam epitaxy. Scientific Reports 2015;5:14760-1-14760-6.

[25] Cheng TS, Davies A, Summerfield A, Cho YJ, Cebula I, Hill RJA, et al. High temperature MBE of graphene on sapphire and hexagonal boron nitride flakes on sapphire J Vac Sci Technol B 2016;34:02L101-1-02L101-6.

[26] Summerfield A, Davies A, Cheng TS, Korolkov VV, Cho YJ, Mellor CJ, et al. Strainengineered graphene grown on hexagonal boron nitride by molecular beam epitaxy. Scientific Reports 2016;6:22440-1-22440-9.

[27] Son M, Lim, H, Hong M, Choi HC. Direct growth of graphene pad on exfoliated hexagonal boron nitride surface. Nanoscale 2011;3:3089-3093.

[28] Yang W, Chen G, Shi Z, Lui C-C, Zhang L, Xie G, et al. Epitaxial growth of single-domain graphene on hexagonal boron nitride. Nat Mater 2013;12:792-797.

[29] Tang S, Wang H, Zhang Y, Li A, Xie H, Lui X, et al. Precisely aligned graphene grown on hexagonal boron nitride by catalyst free chemical vapor deposition. Scientific Reports 2013;3:2666-1-2666-7.

[30] Wang M, Jang SK, Jang W-J, Kim M, Park S-Y, Kim S-W, et al. A platform for large-scale graphene electronics - CVD growth of single-layer graphene on CVD-grown hexagonal boron nitride. Adv Mater 2013;25:2746-2752.

[31] Hwang J, Kim M, Campbell D, Hussain A, Alsalman A, Kwak JY, et al. van der Waals epitaxial growth of graphene on sapphire by chemical vapor deposition without a metal catalyst. ACS Nano 2013;7:385-395.

[32] Lee J-H, Lee EK, Joo W-J, Jang Y, Kim B-S, Lim JY, et al. Wafer-scale growth of single-crystal monolayer graphene on reusable hydrogen-terminated germanium. Science 2014;344:286-289.

[33] Chen J, Guo Y, Jiang L, Xu Z, Huang L, Xue Y, et al. Near-equilibrium chemical vapor deposition of high-quality single-crystal graphene directly on various dielectric substrates. Adv Mater 2014;26:1348-1353.

[34] Tang S, Wang H, Wang HS, Sun Q, Zhang X, Cong C, et al. Silane-catalysed fast growth of large single-crystalline graphene on hexagonal boron nitride. Nat Commun 2015;6:6499-6506.

[35] Hernández-Rodríguez I, García JM, Martín-Gago JA, de Andrés PL, Méndez J. Graphene growth on $\mathrm{Pt}(111)$ and $\mathrm{Au}(111)$ using a MBE carbon solid-source. Diamond and Relat Mater 
$2015 ; 57: 58-62$.

[36] For a review see: Geim AK, Grigorieva IV. Van der Waals heterostructures. Nature 2013;499:419-425.

[37] Koma A. Van der Waals epitaxy - a new epitaxial growth method for a highly latticemismatched system. Thin Solid Films 1992;216:72-76.

[38] Garcia JM, He R, Jiang M.P, Yan J, Pinczuk A, Zuev YM, et al. Multilayer graphene films grown by molecular beam deposition. Solid State Commun 2010;150:809-811.

[39] Dean CR, Young AF, Meric I, Lee C, Wang L, Sorgenfrei S, et al. Boron nitride substrates for high-quality graphene electronics. Nat Nanotechnol 2010;5:722-726.

[40] Tromp R, Rubloff GW, Balk P, LeGoues FK. High-temperature $\mathrm{SiO}_{2}$ decomposition at the $\mathrm{SiO}_{2} / \mathrm{Si}$ interface. Phys Rev Lett 1985;55:2332-2335.

[41] Sasse H-E, König U. SiO diffusion during thermal decomposition of $\mathrm{SiO}_{2}$. J Appl Phys 1990;67:6194-6196.

[42] Song L, Ci L, Lu H, Sorokin PB, Jin C, Ni J, et al. Large scale growth and characterization of atomic hexagonal boron nitride layers. Nano Lett 2010;10:3209-3215.

[43] Eckmann A, Park J, Yang H, Elias D, Mayorov AS, Yu G, et al. Raman fingerprint of aligned graphene/h-BN superlattices. Nano Lett 2013;13:5242-5246.

[44] Wagner J, Ramsteiner M, Wild Ch, Koidl P. Resonant Raman scattering of amorphous carbon and polycrystalline diamond films. Phys Rev B 1989;40:1817-1824.

[45] Park M, Camphausen SM, Myers AF, Barletta PT, Sakhrani V, Bergman L, et al. Raman scattering of tetrahedrally-bonded amorphous carbon deposited at oblique angles. Material Lett 1999;41:229-233.

[46] Ferrari AC, Robertson J. Interpretation of Raman spectra of disordered and amorphous carbon. Phys Rev B 2000;61:14095-14107.

[47] Rebollo BP, Feire Jr FL, Lozada RM, Palomino RM, Jiménez SS, Zelaya OA. Raman shift on n-doped amorphous carbon thin films grown by electron beam evaporation. Phys Status Solidi A 2007;204:964-966.

[48] Ferrari AC. Raman spectroscopy of graphene and graphite: disorder, electron-phonon coupling, doping and nonadiabatic effects. Solid State Commun 2007;143:47-57.

[49] Plaut AS, Wurstbauer U, Pinczuk A, Garcia JM, Pfeiffer LN. Counting molecular-beam grown graphene layers. Appl Phys Lett 2013;102:241905-1-241905-4. 
[50] Koh YK, Bae M-H, Cahill DG, Pop D. Reliably counting atomic planes of few-layer graphene (n > 4). ACS Nano 2011;5:269-274.

[51] Mohiuddin TMG, Lombardo A, Nair RR, bonetti A, Savini G, Jalil R, et al. Uniaxial strain in graphene by Raman spectroscopy: G peak splitting, Grüneisen parameters, and sample orientation. Phys. Rev. B 2009;79:205433-1-205433-8.

[52] Frank O, Tsoukleri G, Parthenios J, Papagelis K, Riaz I, Jalil R, et al. Compression behavior of single-layer graphenes. ACS Nano 2010;4:3131-3138.

[53] Rue S, Maultzsch J, Han MY, Kim P, Brus LE. Raman spectroscopy of lithographically patterned graphene nanoribbons. ACS Nano 2011;5:4123-4130.

[54] Bischoff D, Güttinger J, Dröscher S, Ihn T, Ensslin K, Stampfer C. Raman spectroscopy on etched graphene nanoribbons. J Appl Phys 2011;073710-1-073710-4.

[55] Eckmann A, Felten A, Mishchenko A, Britnell L, Krupke R, Novoselov KS, et al. Probing the nature of defects in graphene by Raman spectroscopy. Nano Lett 2012;12:3925-3930.

[56] Herranz J. Growth by Molecular beam epitaxy of InAs quantum dots on GaAs(001) with site control for their integration in optical microcavities. PhD Thesis, Universidad Complutense de Madrid, (2015) unpublished; and references therein.

[57] Lenski DR, Fuhrer MS. Raman and optical characterisation of multilayer turbostratic graphene grown via chemical vapor deposition. J Appl Phys 2011;110:013720-1-013720-4.

[58] Yan J, Zhang Y, Kim P, Pinczuk A. Electric Field Effect Tuning of Electron-phonon coupling in graphene. Phys Rev Lett 2007;98:166802-1-166802-4.

[59] Das A, Pisana S, Chakraborty B, Piscanec S, Saha SK, Waghmare UV, et al. Monitoring dopants by Raman scattering in an electrochemically top-gated graphene transistor. Nat Nanotechnol 2008;3:210-215.

[60] Malard LM, Pimenta MA, Dresselhaus G, Dresselhaus MS. Raman spectroscopy in graphene. Phys Rep 2009;473:51-87.

[61] Pimenta MA, Dresselhaus G, Dresselhaus MS, Cançado LG, Jorio A, Saito R. Studying disorder in graphite-based systems by Raman spectroscopy. Phys Chem Chem Phys 2007;9:12761290.

[62] Zhao L, He R, Rim KT, Schiros T, Kim KS, Zhou H, et al. Visualizing individual nitrogen dopants in monolayer graphene. Science 2011;333:999-1003.

[63] Wu T, Shen H, Sun L, Cheng B, Lui B, Shen J. Nitrogen and boron doped monolayer 
graphene by chemical vapor deposition using polystyrene, urea and boric acid. New J Chem 2012;36:1385-1391. 
